UVX 2008 (2009) 177-181

(C) EDP Sciences, 2009

DOI: $10.1051 / \mathrm{uvx} / 2009029$

\title{
Characterization and optimization of a flash-X-ray source for diagnostic of dense sprays
}

\author{
E. Romero, C. Cachoncinlle, E. Robert, R. Viladrosa, S. Dozias, \\ G. Coudrat and J.M. Pouvesle
}

GREMI, CNRS/Université d'Orléans, BP. 6744, 45067 Orléans, France

\begin{abstract}
A flash X-ray device dedicated to diagnostic of fuel injection sprays has been developped, tested and improved. Considering the absence of electromagnetic focal system for the electron beam, an important reduction of the X-ray emission zone has been achieved (from $3 \mathrm{~mm}$ to $\sim 150 \mu \mathrm{m}$ ) thanks to carbon fiber cathodes. A full energy characterization, in the range $[5,40] \mathrm{keV}$, has been made with coplanar Germanium detector for 4 types metallic anodes: $\mathrm{Fe}, \mathrm{Cu}, \mathrm{Mo}, \mathrm{W}$.
\end{abstract}

\section{INTRODUCTION}

The diagnosis of near nozzle high speed and dense sprays produced by fuel injection systems needs a fast and reliable technology. However conventional optical non-intrusive methods are clearly not adapted to perform diagnosis of the higher density zone [1] very close to the spray nozzle.

Previous works [2] on $N_{2}$ cryogenic jets in a high pressure chamber had shown that the use of compact flash X-ray system can provide valuable data over a wide range of parameter conditions near the injector orifice. However, in case of fuel injection sprays one has to solve new technical limitations due to the size of the liquid spray which diameter is much smaller than those encountered for cryogenic sprays as radiographied by Metay et al. [2]. A clear radiography of high pressure fuel sprays needs an X-ray spot size comparable to or smaller than the spray diameter $(\sim 140 \mu \mathrm{m})$. In this work we tested different carbon based cathodes with different sizes in order to reduce the X-ray spot size without dose losses.

Another important consideration is the flash X-ray spectral emission. The X-ray transmission patterns of a given thickness of fuel injection spray, and of the same thickness of air (at 50 bar pressure), are very close. This leads to the reduction of the radiography contrast in the image domain of interest. In order to increase the radiography contrast between sprays and air along its path in the chamber volume (combustion chamber), it is needed to select the correct X-ray photon energy band, which is between $6 \mathrm{keV}$ and $10 \mathrm{keV}$. The density measurement of the liquid spray is made through a BeerLambert law inversion, which is extremely difficult to do in cases of polychromatic spectra [3]. To symplify the density calculation, the energy band should be as narrow as possible (monochromatic approach). According to this we compare the spectra obtained with different anodes in the flash X-ray device, in order to choose the material that better matched the selected energy domain for fuel injection spray radiography.

\section{THE FLASH X-RAY SYSTEM}

The flash X-ray (called DIKEV), is a transportable Blumlein table top source build at GREMI laboratory to perform radiography of fuel injection sprays in high pressure ambient gases.

This device delivers high X-ray doses of a few mGy per pulse at the output window of the system in pulses of tens of nanoseconds duration and operates from single shot up to $50 \mathrm{~Hz}$ repetition rate. DIKEV, optimized for charging voltage ranging from 10 to $25 \mathrm{kV}$, leads to the production of X-ray photons with energy ranging from 5 to $40 \mathrm{keV}$. 
This source generates X-ray photons by electron bombardment of a thick metallic target set inside a vacuum electrical isolated chamber called X-ray diode. The electrons are produced by field emission in a pre-arc state of a vacuum discharge. The field emission is induced by the high voltage pulse ranging from 10 to more than $45 \mathrm{kV}$, almost twice the charge voltage (see figure 1), generated by a Blumlein pulse forming line.

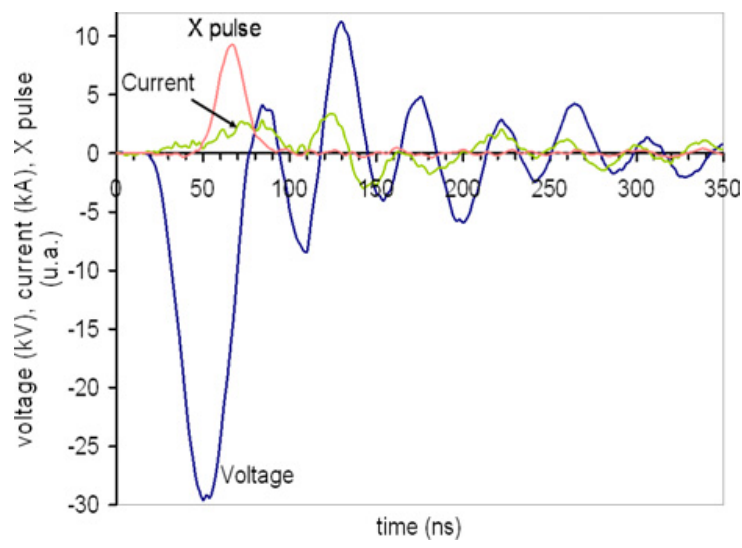

Figure 1. Temporal evolution of $\mathrm{X}$-ray pulse, high voltage pulse and $\mathrm{X}$-ray diode current at $16 \mathrm{kV}$ charging voltage.

\section{X-RAY SPOT SIZE}

The X-ray focus size is not controlled by electrostatic grids as in conventional X-ray tubes. Instead, it is correlated with the pulsed electric field topography in the X-ray diode, which depends on electrodes geometries. The figure 3 shows the electrode geometry used in DIKEV. In all cases, anode was made from a metallic rod and cathode from a carbon base material. Carbon base materials has been selected thank to carbon field emission properties, which is better than metallic materials in a mid vacuum environment [4] and thereby better to produce more stable X-ray pulses. This property of carbon have been observed by direct intensity measures of X-ray pulses in DIKEV 2 like previously by Pouvesle et al. in a close configuration flash X-ray source [5].

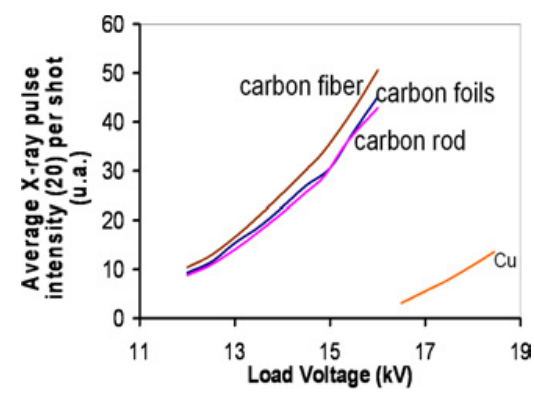

Figure 2. Average X-ray pulse intensity for differents carbon base cathodes and $\mathrm{Cu}$ cathode.

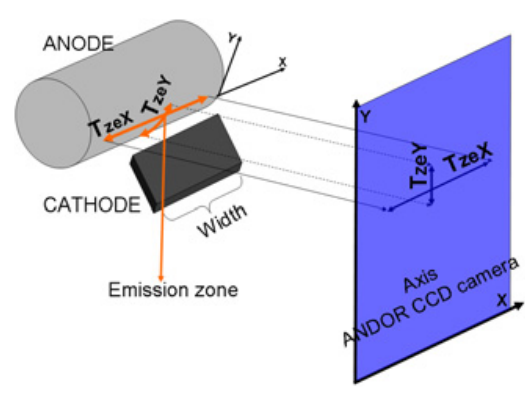

Figure 3. Schematics electrodes geometry.

Three types of carbon based cathodes were tested: carbon rod, carbon foils, and bundle of carbon fiber filaments. We measured the size of X-ray emission zone in the two axis directions: $Y\left(T_{z e Y}\right)$ and $X\left(T_{z e X}\right)$, of the image plane (see figure 3$)$. We tested different carbon foil cathode widths. 
A significant reduction of the X-ray zone emission size was observed when we reduced the cathode width (see figure 4). Tests with cathode width smaller than $0,4 \mathrm{~mm}$ were not performed because of the carbon foils fragility that led in dose reduction and instability. These limitations were also observed with carbon rods of 0,5 and $0,3 \mathrm{~mm}$ diameter.

We were able to reduce the size emission zone (until $T_{z e X} \sim 170 \mu \mathrm{m}$, see figure 5), using carbon fiber filaments in a bundle of $0,3 \mathrm{~mm}$ diameter. This has been possible thanks to the high efficiency of the carbon fiber as field emitter cathode. That allowed an important reduction of cathode size $(10 \mu \mathrm{m}$ diameter each carbon fiber filament) without dose reduction up to 1000 shots.

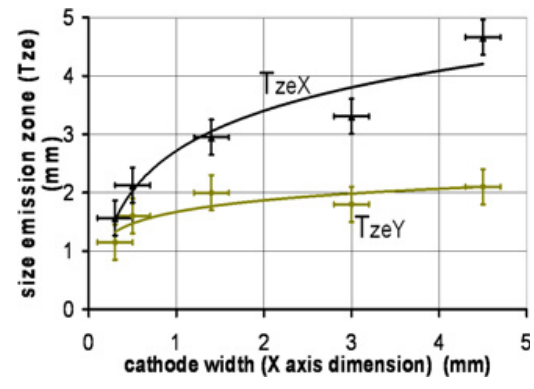

Figure 4. Size reduction of X-ray emission zone, with cathode width reduction.

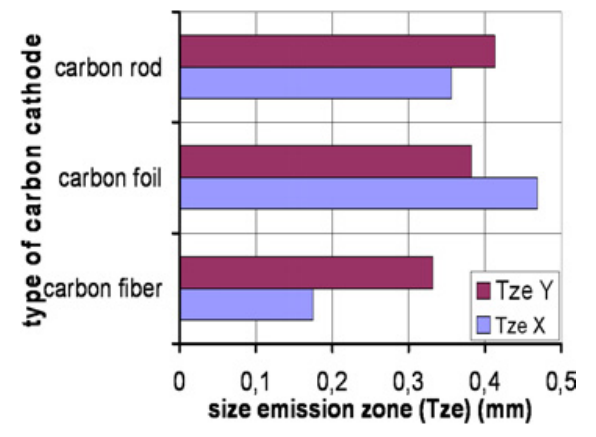

Figure 5. Smaller X-ray emssion zone for different carbon based cathodes.

\section{SPECTRAL CHARACTERIZATION}

The density calculation from Beer Lambert's law requires precise knowledge of X-ray spectrum. For a fuel injection spray in air at 50 bar pressure (combustion chamber), the energy range that guarantees a radiography contrast above a few percent is between $6 \mathrm{keV}$ and $10 \mathrm{keV}$ (at 50 bar pressure).

For four different metallic anodes, a DIKEV full spectra characterization has been made with a Germanium photon detector in a single photon counting mode. The most important $\mathrm{K}$, L characteristics lines below $30 \mathrm{keV}$ for each anode were identified together with Bremsstrahlung spectra. In contrast with Mo anode where continuum spectrum is dominant, a very high intensity ratio between characteristic lines and Bremsstrahlung was measured for $\mathrm{Cu}$ anode ( $\geq 10$, see figure 6 ). The best anode material can be easily selected for a given spray characteristic.

The Germanium detector gives us the total photon number that reaches the surface detector. As we know the experimental acquisition condition, we can calculate the total energy emitted $E_{T X}$ by the flash X-ray source. We estimated the Bremsstralung shape below the characteristic line emissions and fit it with a semi-empirical model to Bremsstrahlung emission generated by electron bombardments on thick solid targets [6]. Counting the total photon number, below the Bremsstralung spectrum, then we calculated the corresponding total Bremsstrahlung emitted energy $E_{\text {brem }}$. Making $E_{T X}-E_{\text {brem }}=E_{\text {lines }}$ we obtain the corresponding total characteristic line emitted energy.

For lower $Z$ anodes $(\mathrm{Cu}$ and $\mathrm{Fe}$ ), a higher characteristic line contribution ( $>60 \%)$ to the $E_{T X}$ has been found. In opposite, for higher $Z$ anodes (W and Mo) Bremsstrahlung was found to represent a large contribution $(>70 \%)$ to the $E_{T X}$. In any case, the lower $Z$ anodes tested are better suited to perform fuel injection spray radiography.

Comparing the total emitted energy $E_{T X}$ with the initial stored energy $E_{T}=0,7 \mathrm{~J}$, in the Blumlein pulse forming line at a charging voltage of $16 \mathrm{kV}$, we obtain an efficiency of $\sim 0,1 \%$, that is quite similar to the efficiency of commercial X-ray tubes. 


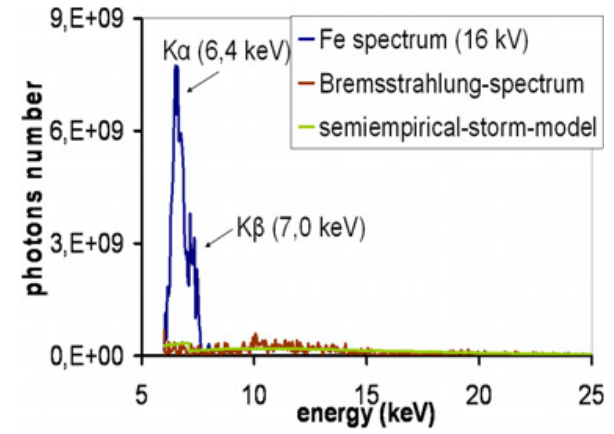

(a) Fe spectra

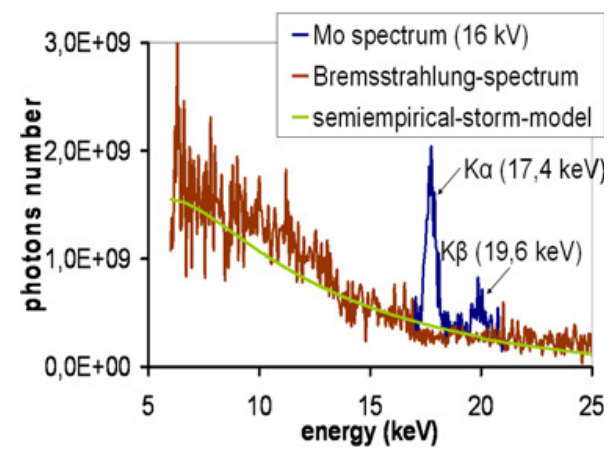

(c) Mo spectra

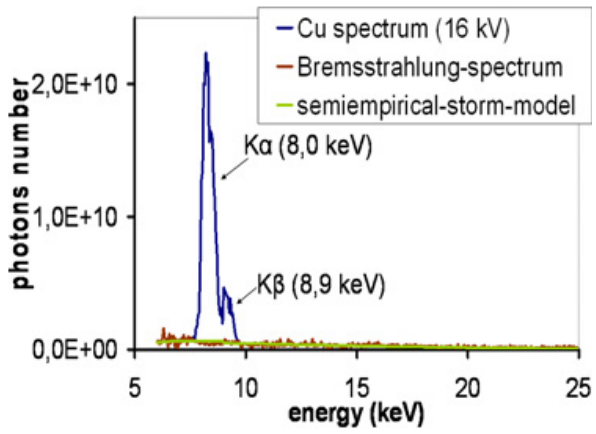

(b) $\mathrm{Cu}$ spectra

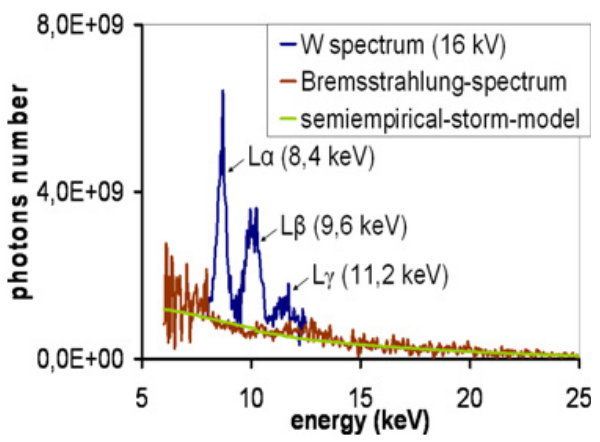

(b) W spectra

Figure 6. Spectra of flash-X-ray at $16 \mathrm{kV}$ of charging voltage.

\section{CONCLUSION}

In this work we reported an important reduction of X-ray spot size, thanks to the carbon fiber cathodes, which produce better quality X-ray pulses.

According to the X-ray transmission pattern through fuel injection spray in a air chamber at 50 bar pressure, copper anode, thanks to a strong characteristics K-line emission, offers the better X-ray spectra to perform fuel injection spray radiographies for density measurements.

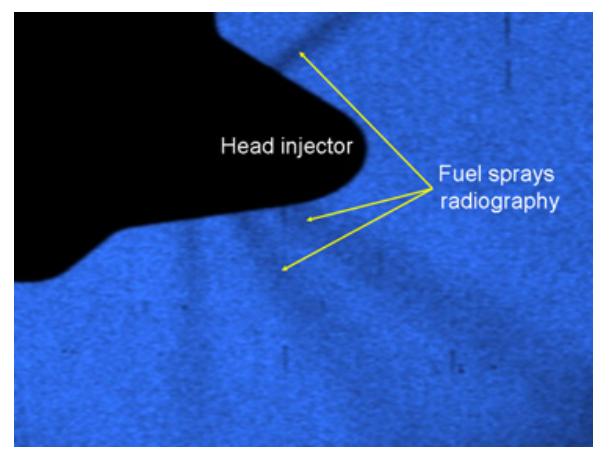

Figure 7. Fuel injection spray radiography. 
As a first step, the energetic radiation group at GREMI laboratory has performed the first high density small diameter spray radiographies in a facility build at GREMI using Cu emission (see figure 7). This is the first radiography of dense spray of light elements in high pressure buffer gas realized with a portable laboratory X-ray source. Ongoing data analysis indicates that spray density in the first millimeter after the nozzle is much smaller than usally expected and used in the models confirming results obtained with synchrotron radiation. More work is presently achieved and will be publised in a near future.

\section{References}

[1] A Kastengren and C F Powell. Spary density measurements using X-ray radiography. Journal Automobile Engineering, 221:653-662, 2007.

[2] Brice Mtay. Diagnostic X par radiographie claire de milieux polyphasiques: Application l'tude de jets cryogniques. $\mathrm{PhD}$ thesis, Universit d'Orlans, 2003.

[3] Pierre Grangeat. La tomographie (fondaments mathmatiques, imagerie microscopique et imagerie industrielle). hermes science, 2002.

[4] V. Filip H. Sugie, M. Tanemura. Carbon nanotubes as electrons source in a x-ray tube. Apply Physics Letter, 78:2878-2580, 2001.

[5] J.M. Pouvesle et al. Compact ash x-ray sources and their applications. Nuclear Instrumental and Methods in Physics Research B, 113:134-140, 1996.

[6] Ellery Storm. Calculated bremsstrahlung spectra from thick tungsten targets. Physical Review A, 5:2328-2338, 1972. 